\title{
INNOVATION HUB ALS NUKLEUS REGIONALER ENTWICKLUNG
}

\author{
Frank Hartmann, Dana Mietzner, Markus Lahr
}

\section{Zusammenfassung}

Das Konzept des Innovation Hubs aufgreifend wird im folgenden Beitrag der Frage nachgegangen, ob sich Innovation Hubs auch außerhalb von Stadtzentren entwickeln können und welche Voraussetzungen hierfür erforderlich sind. Basierend auf empirischen Untersuchungen wird diskutiert, ob geeignete Bedingungen für die Entwicklung eines Innovation Hubs im regionalen Wachstumskern Schönefelder Kreuz gegeben sind, und mittels Szenarien antizipiert wie diese Standortbedingungen in einem zukünftigen Innovation Hub ausgeprägt sein könnten.

\section{Abstract}

Taking up the concept of Innovation Hub, the paper will provide answers to the question whether Innovation Hubs can develop also outside of large cities and which conditions are necessary for this. On the basis of empirical studies the article discusses whether suitable regional conditions for the development of an Innovation Hub within the Regional Growth Core Schönefeld Cross are given and how they can be anticipated using scenario techniques.

\section{»I. FORSCHUNGSANSATZ}

Seit einigen Jahren steht das Thema "Innovation Hub“ auf der Forschungsagenda (vgl. Hall 2001, da Cunha 2007). Der Forschungsansatz beruht wesentlich auf der Symbiose von Konzepten zu räumlich konzentrierten Wissens- und Technologiestandorten (beispielsweise Wissenschafts- und Technologieparks) mit denen der Stadtentwicklung bzw. Stadterneuerung. Damit verbinden sich Fragen der Innovationsforschung, etwa die nach der Bedeutung räumlicher Nähe für die Hervorbringung und Durchsetzung von Innovationen, oder die der Offenheit von Innovationsprozessen (vgl. Chesbrough 2006), mit regionalwissenschaftlichen Fragestellungen. Eine zentrale forschungsleitende These aus regionalwissenschaftlicher Perspektive besteht darin, dass durch die Sanierung entwicklungsbedürftiger Stadtgebiete mittels der Integration von Wissenschaft, Technologie, Kultur, Medien, Design und Kunst die Entwicklung sogenannter intelligenter, innovativer bzw. kreativer Städte forciert werden kann (vgl. da Cunha 2007). Man geht davon aus, dass in solchen Stadtgebieten funktionelle "Schmelztiegel“ entstehen können, die entgegen bisheriger sozialwissenschaftlicher Definitionen nicht nur kulturell durchmischt sind, sondern auch eine Vielzahl an innovativen, parallel existierenden
Funktionen in sich vereinen. Beispielhafte Funktionen sind Forschung und Entwicklung, Kultur, Wohnen und Freizeit. Infolge dessen weisen solche Räume eine hohe Dichte von jungen und hochqualifizierten Bewohnern, ein attraktives räumliches Umfeld sowie ein vielfältiges und interessantes kulturelles Leben auf. Diese multifunktionalen räumlichen Einheiten (Quartiere bzw. Stadtteile) können als mögliche Motoren für die Entwicklung von Stadtregionen angesehen werden, um im globalen wirtschaftlichen Wettbewerb bestehen zu können (vgl. Ache 2000). Sie bieten eine Vielzahl an qualitativ hochwertigen Arbeitsplätzen und stellen gleichzeig einen attraktiven Lebensraum dar, durch den Arbeitskräfte längerfristig an den Standort gebunden werden können.

Fasst man die bisherigen Forschungsarbeiten zusammen und bringt die Sicht der regionalorientierten Innovationsforschung in eine Arbeitsdefinition ein (vgl. Hartmann und Große 2012), lässt sich ein Innovation Hub als ein räumlich konzentrierter Standort zur Hervorbringung und Vermarktung von Innovationen beschreiben, die durch das aufeinander bezogene Agieren von Wissenschaft, Bildung und Wirtschaft in Kombination mit passfähigen Wohnbedingungen sowie Kultur-, Shoppingund Unterhaltungsangeboten befördert werden. Ein Innovation Hub ist folglich durch eine Vielzahl von Interaktionen zwischen den entsprechenden Akteuren gekennzeichnet und die Grenzen zwischen physischen, digitalen, ökonomischen, sozialen und kulturellen Räumen werden in ihm aufgehoben. Es bilden sich kreative Communities mit einer großen Dichte an Wissensarbeitern, die eine hohe Lebensqualität suchen, einschließlich sozialer und kultureller Vielfalt sowie digitaler und physischer Erreichbarkeit (vgl. Hall 2001 und da Cunha 2007).

Besonders geeignete Bedingungen für Innovationen entwickeln sich in Stadtregionen, demzufolge durch eine spezifische Integration der oben genannten Funktionen. Wenn dem so ist, und dafür sprechen zahlreiche empirische Befunde (vgl. Florida 2005), und wenn der generelle Trend der Suburbanisierung noch länger anhält (vgl. Mädig 2004, Köppen 2008), stellt sich die Frage, ob sich Innovation Hubs nicht nur in den Zentren großer Metropolen entwickeln können, sondern auch in suburbanen Räumen bzw. peripheren Edge Cities (Garreau 1991).

Dieser Fragestellung wurde im Zuge der Anwendung des Konzepts auf eine konkrete Region nachgegangen. Die Stadt Königs Wusterhausen beauftrage im Jahr 2011 die TH Wildau $[\mathrm{FH}]$ zu prüfen, ob begründete Chancen dafür bestehen, ausgehend 
von der Entwicklungsdynamik des ehemaligen Schwermaschinenbau-Geländes in Wildau, mit seinen Technologiezentren, Unternehmen und dem Campus der TH Wildau, ein Teilareal des Funkerberges in Königs Wusterhausen zu einem technologisch orientierten Anpark zu entwickeln. Bei dieser Untersuchung wurde von den Autoren die These zugrundegelegt, dass der Funkerberg Bestandteil der integrativen Entwicklung einer umfassenderen Teilregion des Regionalen Wachstumskerns Schönefelder Kreuz sein sollte, die zur Herausbildung eines Innovation Hubs führen könnte (vgl. Abbildung 1). Die Besonderheit eines solchen Innovation Hubs bestünde darin, dass er sich nicht in einer größeren Stadt oder Metropole entwickeln würde, sondern im suburbanen Raum einer Metropole und inmitten einer sich profilierenden Flughafenumfeldregion. Ein solcher Hub könnte sich zur Drehscheibe auf einer der zentralen
Entwicklungsachsen der Flughafenumfeldregion Berlin-Brandenburg entwickeln, die von Ludwigsfelde über Dahlewitz und den Flughafen in Schönefeld bis hinein in die Metropole Berlin führt.

\section{» II. BEDINGUNGEN FÜR DIE ENTWICKLUNG EINES INNOVATION HUBS}

Um diese Entwicklungschancen auszuloten, wurden in der bisherigen Literatur zu Innovation Hubs herausgearbeitete zentrale Entwicklungsbedingungen (vgl. da Cunha 2007) mit gängigen "harten" und "weichen" Standortfaktoren (vgl. Meyer-Stamer 1999) abgeglichen und ein Faktorenset erstellt (32 Faktoren). Dieses Faktorenset wurde inhaltlich weiter untersetzt und durch ein internes Team der Projektbearbeiter, durch Vertreter der Wirtschaftsförderung der Stadt
Königs Wusterhausen und der Gemeinde Wildau, Vertreter des Arbeitskreises Funkerberg, der Wohnungswirtschaft Wildau, von ausgewählten regional ansässigen Unternehmen sowie weiteren Mitarbeitern der TH Wildau als Stärken und Schwächen sowie Chancen und Risiken bewertet. Insgesamt gingen die Wertungen von 14 externen Experten in die Bewertung ein.

Im Ergebnis lässt sich feststellen, dass die überwiegende Zahl der untersuchten 32 Standortfaktoren, die den Bereichen (1) Wirtschaft, (2) Architektur und bauliches Umfeld, (3) Naturraum und räumliche Lage, (4) Kultur und öffentlicher Raum, (5) Verkehrs- und Dateninfrastruktur, (6) Arbeitsmarkt und Sozialstruktur/Bildung zugeordnet wurden, nach Auffassung von Experten und zugrundeliegenden Studien bereits gegenwärtig gut ausgeprägt sind. Es konnten zahlreiche Stärken und Chancen identifiziert werden, was für die erfolgreiche Etablierung eines Innovation

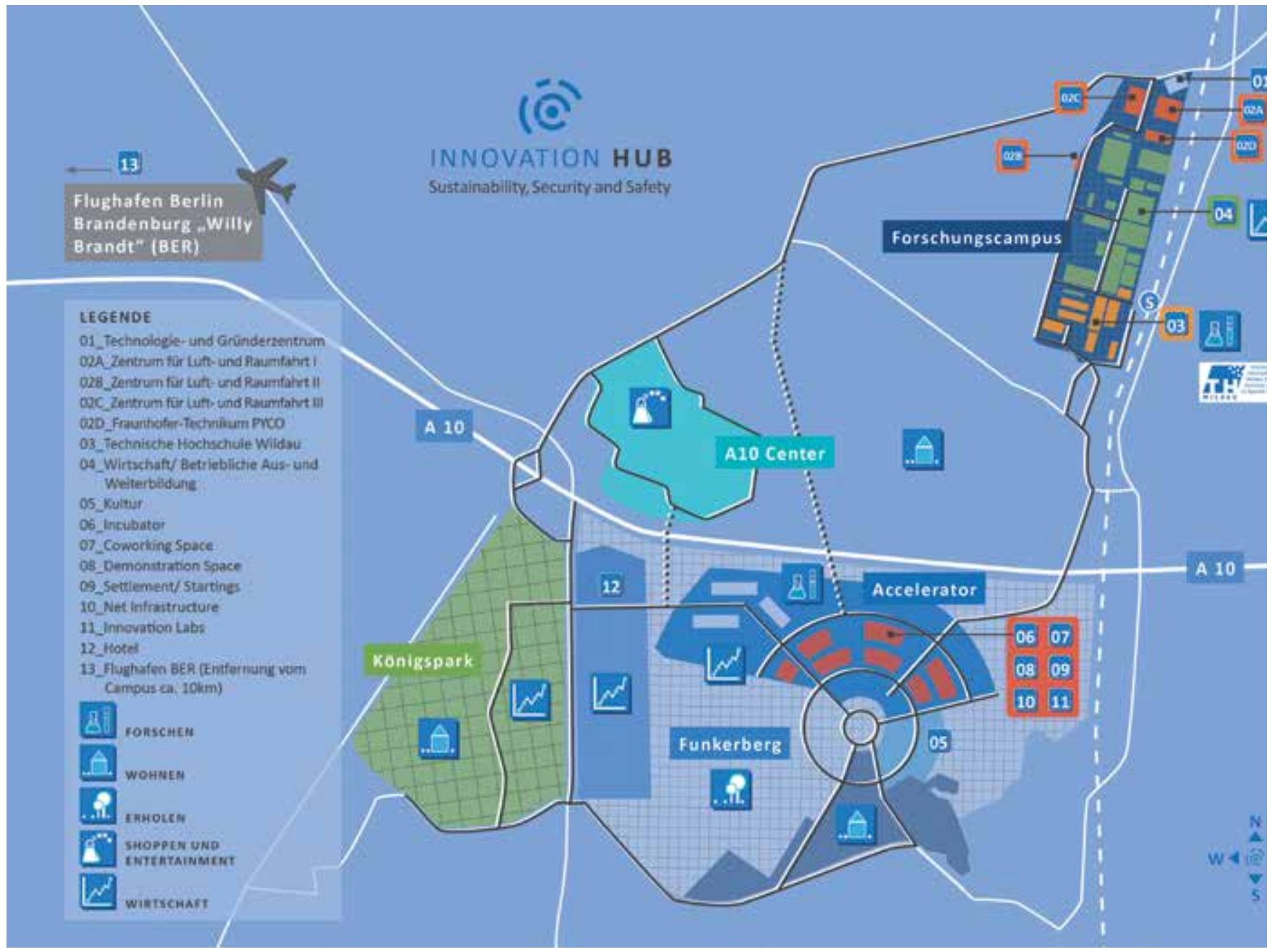


Hubs Funkerberg spricht. Besonders positiv ausgeprägt sind die Standortfaktoren "Ansiedlungsgeschehen“, „Verkehrsinfrastruktur“, „Rolle der Technologie- und Gründerzentren“ sowie „Nähe zu Forschung und Entwicklung“. Allerdings gibt es auch Faktoren, die als nachteilig ausgeprägt bewertet wurden. Zu diesen Faktoren gehören (1) die Identifikation der lokalen Bevölkerung mit einem Innovation Hub sowie ein diesbezügliches Image. Des Weiteren wurden (2) die Multikulturalität, als Ausdruck einer offenen und international orientierten Region, (3) der Lifestyle, im Sinne eines urbanen Lebensstiles, und (4) die Emissionsbelastungen infolge der verkehrstechnischen Einbindung des Standortes als nachteilig ausgeprägt eingeschätzt. Auch der Standortfaktor Governance, als kooperative und zugleich integrierende Form regionaler politischer Gestaltung, muss im Hinblick auf die zügige und entschlossene Entwicklung eines Innovation Hubs Funkerberg gezielt weiterentwickelt werden.

\section{»III. ERARBEITUNG VON SZENARIEN}

Aufbauend auf der Konzeptentwicklung zu Innovation Hubs sowie der Analyse und Bewertung förderlicher Entwicklungsbedingungen wurden Szenarien mit dem Zukunftshorizont 2027 entwickelt. Das Ziel der Szenarioanalyse bestand in der Herausarbeitung unterschiedlicher Zukunftsbilder zur Entwicklung des Innovation Hubs Funkerberg. Durch die systematische Entwicklung von Szenarien sollte aufgezeigt werden, wie der Innovation Hub Funkerberg ausgestaltet werden kann und welche Faktoren seine Entwicklung befördern oder hemmen können.

Der Prozess der Szenarioanalyse beginnt generell mit einer systematischen Bestandsaufnahme. Darauf aufbauend werden Einflussfaktoren abgeleitet und in einer Einflussanalyse näher untersucht, mit dem Ziel Schlüsselfaktoren zu identifizieren, welche die zukünftigen Entwicklungsmöglichkeiten des Szenariofeldes signifikant beschreiben.
In einem weiteren Schritt schließt sich die Ableitung von Zukunftsprojektionen an, die dann mithilfe einer Konsistenzanalyse zu Szenarien gebündelt werden. Abschließend werden Szenarien beschrieben ( $\mathrm{vgl}$. Gausemeier et al. 1996, Mietzner 2009).

In der Konzeptstudie zum Innovation Hub Funkerberg dienten die identifizierten und bewerteten Standortfaktoren zur erweiterten Beschreibung der Ausgangssituation für die Entwicklung eines Innovation Hubs und wurden im Prozess der Erarbeitung von Szenarien einer Einflussanalyse unterzogen. Zu diesem Zweck wurden die 32 Faktoren durch das Bearbeitungsteam in mehreren Runden zueinander in Beziehung gesetzt und die Stärke der Beziehungen zwischen den Faktoren eingeschätzt. Die Auswertung der Vernetzung der Faktoren (Aktivsummen und Passivsummen) führte zu einem Systemgrid (vgl. Abb. 2), in dem die Faktoren in Abhängigkeit vom Grad ihrer Einflussnahme und in

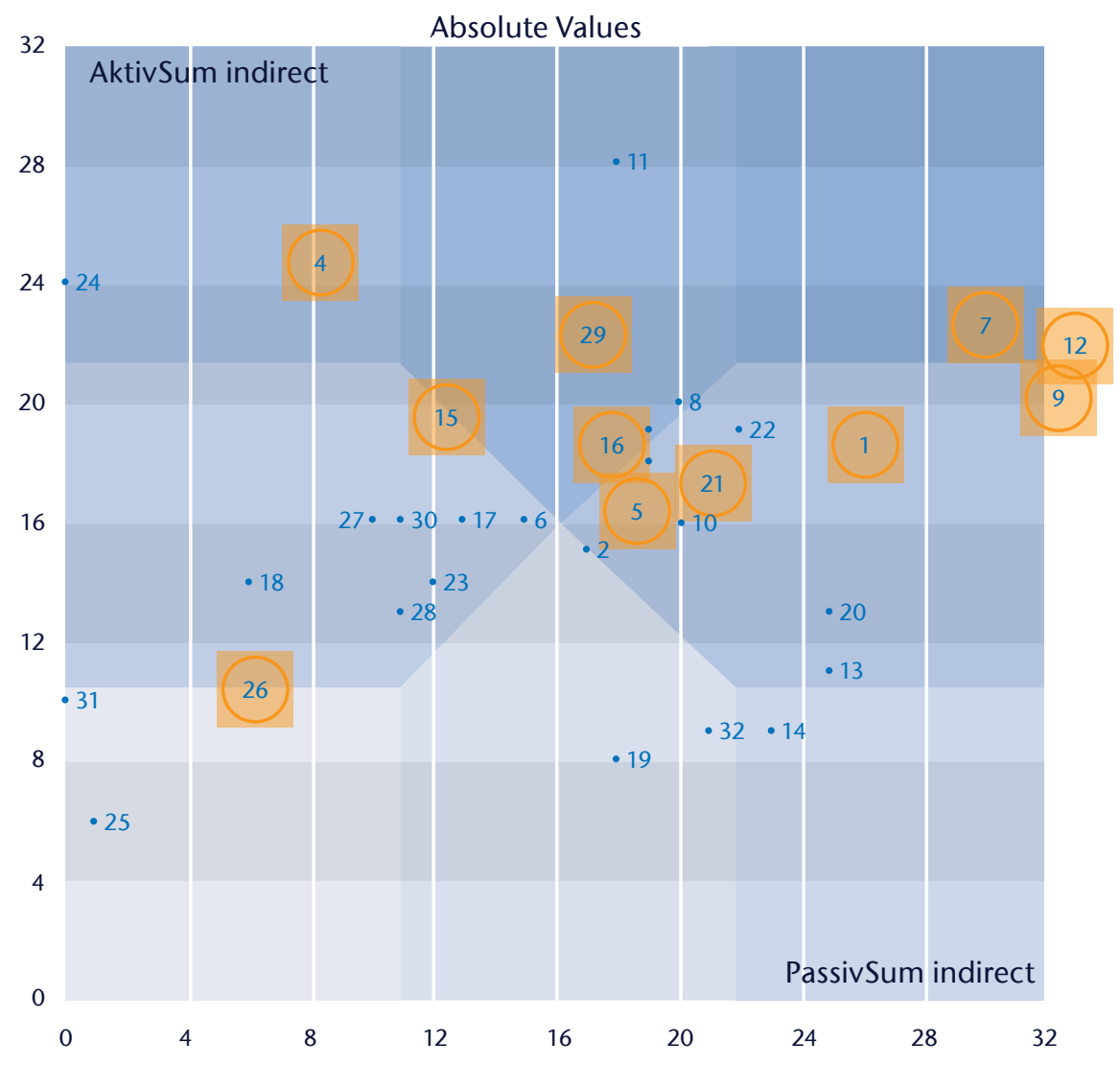

Abb. 2) Systemgrid für die vernetzten Standortfaktoren

\section{EINFLUSSFAKTOREN:}

1 Grad ökonomischer Spezialisierung

2 Rolle Kreativwirtschaft

3 Entrepreneurship Culture

4 Nähe zu FuE

5 Institutionelle Kooperation Netzwerke

6 Rolle TGZ

7 Wirtschaftsstruktur

8 Verfügbare Flächen

9 Ansiedlungsgeschehen

10 Expansionsdruck ansässiger Firmen

11 Bildung

12 Fachkräfte

13 Standortbindung von Studenten

14 Sozialstruktur

15 Multikulturalität

16 Verbindung Wohnen Arbeiten Studieren Freizeit

17 Architektur

18 Raumentwicklungskonzept

19 Gestaltung des öffentlichen Raumes 20 Identität/Image

21 Kultureinrichtungen Angebote

22 Lifestyle Dynamik

23 Umwelt und Landschaftsqualität

24 Räumliche Lage

25 Landschaftsbesonderheiten

26 Emissionen

27 Verkehrsanbindung

28 Digitale Infrastruktur

29 Regionale Entwicklungsstrategie

30 Politisch-administratives Handeln

31 public-private-partnership

32 Langfristige Projektunterstützung 
Abhängigkeit vom Grad ihrer Beeinflussbarkeit geclustert wurden. Die zur Berechnung eingesetzte Software ${ }^{1}$ berücksichtigt darüber hinaus auch indirekte Beziehungen zwischen den Faktoren. Entsprechend der Positionierung der Faktoren in den Quadranten der Matrix werden Systemhebel, proaktive Knoten, interaktive Knoten, reaktive und proaktive Knoten, Systemindikatoren, reaktive Puffer und unabhängige Puffer unterschieden. Während besonders aktive Faktoren eine größere Zahl anderer Faktoren relativ stark beeinflussen und auch als Systemhebel bezeichnet werden können, werden die interaktiven Knoten gleichzeitig von anderen Faktoren stark beeinflusst. Sie haben großen Einfluss auf das Gesamtsystem, weil sie in dieses sehr stark eingebunden sind. Passive Faktoren werden in hohem Maße von anderen Faktoren beeinflusst, ohne diese in starkem Maße zu beeinflussen. Aus diesem Grund verweisen sie auf mögliche Systemänderungen und eignen sich besonders als Frühwarnindikatoren. Die reaktiven und unabhängigen Puffer sind nur wenig in das System eingebunden und werden in Szenariobildungsprozessen häufig ausgeklammert. Für die Erarbeitung von Szenarien sind somit insbesondere die
Systemhebel sowie die interaktiven und proaktiven Knoten geeignet.

Im daran anschließenden Analyseschritt wurden für jeden ermittelten Schlüsselfaktor Projektionen, d. h. mehrere alternative Entwicklungsmöglichkeiten, beschrieben. Diese Zukunftsprojektionen sind in der Regel keine quantitativen Prognosen, sondern qualitative Beschreibungen, mit denen sich zukünftige, mögliche Entwicklungen verdeutlichen lassen. Die tatsächliche Szenario-Bildung beginnt mit der Bewertung der Verträglichkeit der einzelnen Zukunftsprojektionen im Rahmen einer Konsistenzanalyse. In diesem Schritt wird untersucht, ob zwei Projektionen in Zukunft zusammen auftreten können. Durch unterschiedliche Annahmen über die Zukunftsentwicklung der treibenden Faktoren können so verschiedene Szenarien entwickelt werden. Es entstehen differenzierte, in sich konsistente Szenarien. Diese werden analysiert, interpretiert und in einer kommunikationsfähigen Form beschrieben.

Im Fallbeispiel wurden für die 11 Schlüsselfaktoren insgesamt 28 Projektionen erarbeitet und auf ihre Konsistenz überprüft. Im Anschluss an die Konsistenzprüfung erfolgte unter Zuhilfenahme des Softwaretools die Auswahl geeigneter Projektionsbündel als Basis für die entsprechenden Szenarien. Auf der Basis der softwaregestützten Konsistenzanalyse konnten drei Szenariovorschläge ermittelt werden. Das erste Szenario beinhaltete die Alleinstellungsmerkmale "Hoher Grad ökonomischer Spezialisierung“ und "innovative und integrierende regionale Entwicklungsstrategie“, das zweite Szenario das zentrale Projektionsbündel „Attraktionsstandort" und das dritte Szenario die zentralen Projektionsbündeln "Profilbegrenzung“ und "Schrumpfende Wirtschaft".

Die Clusterung der Szenariovorschläge und deren interne Konsistenz zeigt $\mathrm{Ab}$ bildung 3. Hier wird sichtbar, dass die Szenarien 1 und 3 sehr stark geclustert und in sich „stimmig“ sind. Das Szenario 2 weicht hiervon etwas ab und ist nicht in diesem hohen Maße konsistent und wurde demzufolge nicht ausformuliert.

Das erste Szenario „Innovation Hub Funkerberg als Motor der Flughafenumfeldregion" beschreibt eine positive Zukunftsentwicklung ausgewählter Schlüsselfaktoren. Das Szenario ist

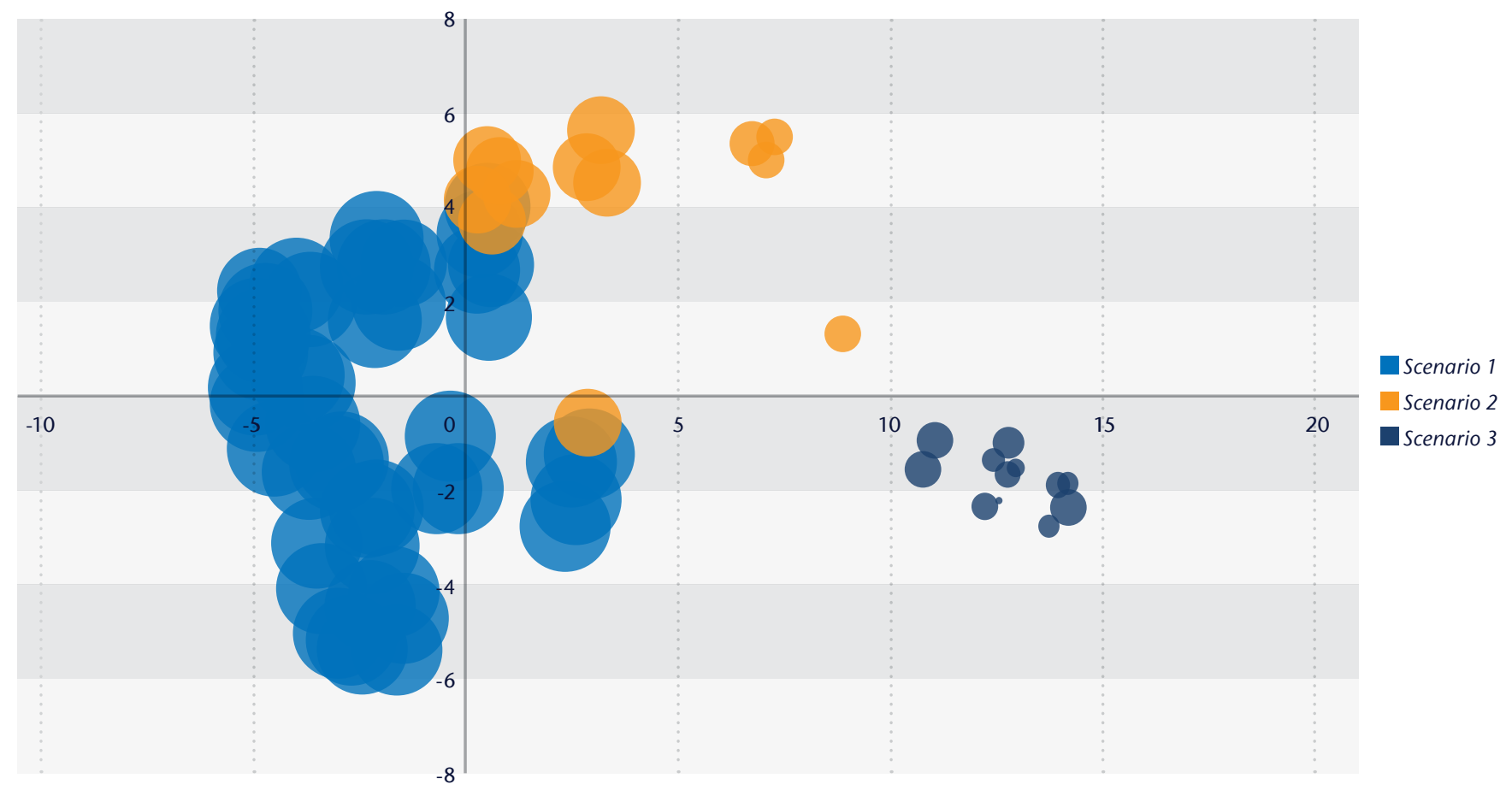

$A b b .3)$ Clusterung der Szenarien im Ergebnis der Konsistenzanalyse 
durch eine deutliche Profilbildung des Innovation Hubs Funkerberg und eine vorteilhafte Entwicklung wichtiger Standortfaktoren charakterisiert. Im zweiten Szenario „Zufallsgetriebene Spezialisierung und Profilbegrenzung im Innovation Hub Funkerberg" mangelt es deutlich an einer klaren Profilbildung des Innovation Hubs sowie einer adäquaten Entwicklung weiterer zentraler Standortfaktoren. Zudem haben in diesem Szenario die dynamischen Entwicklungen an anderen Standorten die verzögerte Entwicklung des Innovation Hubs Funkerberg schlichtweg überrollt.

\section{»IV. SCHLUSSFOLGERUNGEN}

Das Fallbeispiel belegt die These, dass sich Innovation Hubs auch außerhalb von großen Städten oder Metropolen entwickeln können. Voraussetzung dafür ist, dass an solchen Orten kritische Massen an Innovationspotenzialen entstehen, diese Potenziale sich in überregionale Innovationsstrukturen (Cluster) und Raumkontexte (Flughafenumfeldregion oder Metropolenregion) einbinden und die erforderlichen Standortbedingungen zielgerichtet auf hohem Niveau entwickelt werden. Solche Innovation Hubs können dann, so die Hypothese weiter konkretisiert, hochgradig spezifische Komponenten und Funktionen ausbilden wie beispielsweise den Betrieb eines Inkubators, das Vorhalten von Coworking Space, den Betrieb von Innovation und Living Labs, die Bereitstellung und den Betrieb von Demonstrationsflächen für innovative Entwicklungen oder von gemeinsamen Laborflächen und anderer Infrastruktureinrichtungen für Wissenschaft und Wirtschaft.
Ache, P. (2000): Was treibt den Motor an? Stadtregionen zwischen innovativen Milieus und Urban Governance. Raumforschung und Raumordnung 58(2-3), 244-253.

Chesbrough, H. W., Vanhaverbeke, W., West, J. (2006): Open innovation: Researching a new paradigm. Oxford: Oxford University Press.

da Cunha, V. (2007): Creative Urban Regeneration: The Case of 'Innovation Hubs'. In: International Journal of Innovation and Regional Development (Vol 1 No. 4).

Florida, R. L. (2005): The flight of the creative class: The new global competition for talent. New York: Harper Business.

Garreau, J. (1991): Edge City. Life on the New Frontier.

Gausemeier, J., Fink, A., Schlake, O. (1996): SzenarioManagement: Planen und Führen mit Szenarien. 2. Aufl. München, Wien: Hanser.

Hall, P. (2001): Cities in Civilization: Culture, Innovation and Urban Order. London: Weidenfeld\&Nicholson.

Hartmann, F., Große, U. (2012): Innovation Competence in Regions: A Case Study Approach: XXII ISPIM Conference, Barcelona, paper accepted for presentation.

Köppen, B. (2008): Reurbanisierung als Hoffnung der Städte im demographischen Wandel? In: Maretzke, St. (Hrsg.) Städte im demographischen Wandel. Materialien zur Bevölkerungswissenschaft, Heft 125, Wiesbaden, $31-40$

Mädig, H. (2004): Raus aus der Stadt? Zur Erklärung und Beurteilung der Suburbanisierung. DifU Berichte 2004.

Meyer-Stamer, J. (1999): Lokale und regionale Standortpolitik - Konzepte und Instrumente jenseits von Industriepolitik und traditioneller Wirtschaftsförderung. Duisburg. (INEF-Report, 39).

Mietzner, D. (2009): Strategische Vorausschau und Szenarioanalysen: Methodenevaluation und neue Ansätze: Gabler Verlag 2009.

\section{AUTOREN}

Frank Hartmann

frank.hartmann@th-wildau.de

Dana Mietzner

dana.mietzner@th-wildau.de

Markus Lahr

markus.lahr@th-wildau.de

Forschungsgruppe Innovations- und Regionalforschung, TH Wildau [FH] 